[This version is a preprint. The final version will appear in History and Philosophy of the Life Sciences, https://doi.org/10.1007/s40656-020-00305-2]

Javier Suárez $^{12}$

Contact Details: javier.suarez@ub.edu

\title{
“The stability of traits conception of the hologenome: An evolutionary account of holobiont individuality"
}

Bourrat and Griffiths (HPLS 40(2): 33, 2018) have recently argued that most of the evidence presented by holobiont defenders to support the thesis that holobionts are evolutionary individuals is not to the point and is not even adequate to discriminate multispecies evolutionary individuals from other multispecies assemblages that would not be considered evolutionary individuals by most holobiont defenders. They further argue that an adequate criterion to distinguish the two categories rests on the existence of fitness alignment, presenting the notion of fitness boundedness as a criterion that allows divorcing true multispecies evolutionary individuals from other multispecies assemblages and provides an adequate criterion to single out genuine evolutionary multispecies assemblages. A consequence of their criterion is that holobionts, as conventionally defined by hologenome defenders, are not evolutionary individuals except in very rare cases, and for very specific host-symbiont associations. This paper is a critical response to Bourrat and Griffiths' arguments and as such it constitutes a refinement and a defence of the arguments presented by holobiont defenders. Drawing upon the case of the hologenomic basis of the evolution of sanguivory in vampire bats (Nat. Ecol. Evol. 2: 659-668, 2018), I argue that Bourrat and Griffiths overlook some aspects of the biological nature of the microbiome that justifies the thesis that holobionts are evolutionarily different to other multispecies assemblages. I argue that the hologenome theory of evolution should not define the hologenome as a collection of genomes, but as the sum of the host genome plus some traits of the microbiome which together constitute an evolutionary individual, a conception I refer to as the stability of traits conception of the hologenome. Based on that conception I argue that the evidence presented by holobiont defenders, if adequately refined under the "stability of traits" framework I advocate here, is to the point, and supports the thesis that holobionts are evolutionary individuals. In this sense, the paper offers an account of the holobiont that aims to foster a dialogue between hologenome advocates and hologenome critics.

\footnotetext{
${ }^{1}$ Logos, BIAP / University of Barcelona (Spain).

${ }^{2}$ Egenis: The Centre for the Study of Life Sciences, University of Exeter (UK).
} 
Keywords: Holobiont; Biological individuality; Evolutionary individuality; Symbiosis; Fitness alignment; Hologenome; Microbiome; Stability of traits

\section{Introduction}

Holobionts are biological assemblages composed by a multicellular host, or macrobe, ${ }^{3}$ plus its symbiotic microbiome. The term was originally coined by Lynn Margulis to refer to the eukaryotic cell, as well as to other examples of vertically inherited symbioses (e.g. aphid-Buchnera aphidicola), where the connection between the partners was so tight that they could be conceived as a physiological and evolutionary unit (1990, 1991; see O’Malley 2017; Suárez 2018). Margulis’ original idea was that these symbiotic pair associations ${ }^{4}$ were so intimate and the individuals were so closely related to each other since the beginning of their life that they should be better understood by analogy with a zygote -i.e. a fusion of independent cell lineages that formed a new individual- than as two independent individuals.

After Margulis introduced the term in the early 90s, it remained largely unused until Reshef et al. (2006) formulated the coral probiotic hypothesis and first referred to corals as holobionts. ${ }^{5}$ The coral probiotic hypothesis is a theory about the individuality of corals. It is used to account for the loss of the bacteria Vibrio shiloi in most of the coral samples of the species Oculina patagonica studied. In the 90s, several studies on coral bleaching had reported that $V$. shiloi was the agent responsible for coral bleaching (Kushmaro et al. 1997, 2001). However, several studies conducted in the early 2000s reported that many of the corals previously infected by $V$. shiloi had overcome the infection. This fact created an important puzzle since corals are known to lack an adaptive immune system. If they lack an adaptive immune system, then every possible mechanism of defence against pathogens can only evolve on an evolutionary timescale and, therefore, how corals got rid of $V$. shiloi remains largely mysterious. The coral probiotic hypothesis posits that corals should be conceived as holobionts, integrated units composed by the $O$. patagonica plus its symbionts. In this vein, the elimination of $V$. shiloi in the coral samples could be explained as the result of the interactions within the coral microbiome, which would have created the conditions that had made it possible.

\footnotetext{
${ }^{3}$ Following Dupré and O’Malley (2009), I will use the term “macrobe” or “microorganism” to refer to the mono-zygotically-descended multicellular host that composes the holobiont.

${ }^{4}$ Not necessarily pair associations. See e.g. Margulis and Sagan (2001).

${ }^{5}$ There is a previous use in a public lecture by Jefferson (1994), and in a paper about corals by Rohwer and colleagues (2002: 8, Fig. 5). However, Jefferson did not keep developing the theory, and Rohwer et al. only introduced the concept in a figure. Furthermore, contrary to what happened with the case of Reshef and colleagues, in neither of these cases was the term used immediately afterwards to open a debate about the concept of biological individuality.
} 
Two years later, Zilber-Rosenberg and Rosenberg (2008), departing from the observation that all animals and plants engage in symbiotic relationships with abundant microorganisms, generalized the use of "holobiont" to every macrobe. Furthermore, they coined the concept of "hologenome" to refer to the sum of the genome of the host and the genomes of its microbes, and formulated the "hologenome theory of evolution" (HTE, hereafter). ${ }^{6}$ It is important to note, although in passing, that the authors who have been using the term "holobiont" since Zilber-Rosenberg and Rosenberg formulated HTE are expressing a different holobiont concept than the concept that was coined by Margulis in the 90s: whereas the latter referred to vertically inherited host-symbiont associations, the former referred to the association between a host plus all its symbiotic microbiome (Suárez 2019: 27-44). In this paper, I will use "holobiont” and "hologenome" as it is used after the formulation of HTE. ${ }^{7}$

HTE is a theory about the individuality of macrobes that consists in a series of different, although interrelated theses ${ }^{8}$ : (1) Holobionts are anatomically integrated units; (2) holobionts are physiologically integrated individuals; (3) holobionts are developmental units; (4) holobionts are immunological individuals; (5) holobionts are evolutionary units (units of selection) ${ }^{9}$ (Gilbert et al. 2012, 2017; Rosenberg and ZilberRosenberg 2013; Bordeinstein and Theis 2015; Theis et al. 2016; Lloyd 2017b;

Roughgarden et al. 2018). There are different theories about which of the theses are true of holobionts, since each is supported differently by the empirical evidence currently available. Among all, the thesis that holobionts are units of selection has probably been the most contested among philosophers of biology and, as many authors tend to equate "biological individual” with “unit of selection” (e.g. Godfrey-Smith 2009; Clarke 2013), its confutation has been used to argue that holobionts are not biological individuals (Moran and Sloan 2015; Godfrey-Smith 2015; Douglas and Werren 2016; Skillings 2016; Hurst 2017; Stencel and Wloch-Salamon 2018).

\footnotetext{
${ }^{6}$ Sometimes also called the "hologenome concept of evolution" (Rosenberg and Zilber-Rosenberg 2013, 2016).

${ }^{7}$ In fact, it is likely that HTE would not be as controversial if the concept of holobiont kept its original meaning, as it was first formulated by Margulis (Díaz 2015).

${ }^{8}$ Most of these theses have been developed and clarified after HTE was first formulated, so the reader might not interpret this paragraph as meaning that all those theses were already clearly expressed in Zilber-Rosenberg and Rosenberg (2008). These theses correspond to different conceptual developments on the study of biological individuality and its application to the study of hologenomes (e.g. Dupré and O’Malley 2009; Dupré 2010, 2012, 2017; Pradeu 2016; DiFrisco 2017; Lidgard and Nyhart 2017).

${ }^{9}$ Strictly speaking, it is not accurate to identify evolutionary individuals with units of selection, since there can be units that might be argued to evolve -e.g. by neutral evolution, or by processes of sortingdespite not being naturally selected (e.g. Vrba and Gould 1986; Maynard-Smith 1987, 1991; Okasha 2006). In a strict sense, being a unit of selection is only necessary for cumulative evolution that leads to adaptations, but not for evolution per se (Suárez and Triviño 2020). Nonetheless, as the two notions are commonly used interchangeably, I will use them interchangeably in this paper as well.
} 
Following the contemporary discussions about the status of holobionts as units of selection, Bourrat and Griffiths (HPLS 40(2): 33, 2018) have recently argued that the evidence presented by HTE defenders to support the thesis that holobionts are evolutionary individuals -thesis (5) from above- is simply not to the point, and does not allow to convincingly distinguish between holobionts and other multispecies assemblages that would not be considered evolutionary individuals by holobiont defenders. To overcome this difficulty, Bourrat and Griffiths develop a criterion of fitness boundedness that, they argue, allows to distinguish between true multispecies evolutionary individuals and other multispecies assemblages. Although I share some of Bourrat and Griffiths' general concerns about the soundness of the arguments in favour of HTE, especially when these concern part of the system arguments (see section 2), I think part of our evidence about the nature of the microbiome can be used to support the claim that holobionts are evolutionary individuals and are different from other multispecies assemblages, if this evidence is adequately interpreted. In that vein, in this paper, I contest Bourrat and Griffiths' arguments and defend that the original evidence that defenders of HTE have presented to argue that holobionts are evolutionary individuals is to the point and, indeed, satisfies Bourrat and Griffiths' criterion for evolutionary individuality if it is interpreted according to the concept of stability of traits or trait-recurrence (Suárez and Triviño 2019; Suárez 2019). Interpreted this way, the part of the system arguments adduced by HTE defenders recover their force, and thus it makes sense to argue that holobionts are evolutionary individuals. To prove so, I draw on the case of the hologenomic adaptations that underlie the evolution of sanguivory in vampire bats (Mendoza et al. 2018). Based on it, I argue that the biological nature of the microbiome justifies the consideration of the holobiont as an evolutionary individual in a manner that does not equally apply to other multispecies assemblages. My position, thus, can be seen as a refinement of the part of the system arguments originally proposed by HTE advocates that makes the hologenome compatible with contemporary biological knowledge.

My agenda will be as follows: First, I present Bourrat and Griffiths' criticisms against the arguments presented by defenders of HTE (section 2.1), as well as their constructive proposal of fitness boundedness, which they use to distinguish multispecies evolutionary individuals form other multispecies assemblages (section 2.2). In section 3, I present some current research about the hologenomic evolution of vampire bats, and show how the part of the system arguments used by HTE defenders do indeed mask some information about the biological properties of the holobiont that are evolutionary in nature. Drawing on that, in section 4 I present an evolutionary account of the holobiont that I call "stability of traits" account, and according to which (1) the evolutionary claims masked in the part of the system arguments concern the notion of fitness boundedness introduced by Bourrat and Griffiths; (2) contra Bourrat and Griffiths, I argue that the fitness boundedness in the holobiont should not be looked for in the lineages or taxa that compose the host's microbiome, but in its traits (genes, or 
genetic components). ${ }^{10}$ In that vein, I redefine HTE as a theory about the evolution of the host genome plus some specific traits of its microbiome, rather than as the sum of genomes that compose a holobiont. I use this last point to distinguish my proposal from other claims about the role of the holobiont as an evolutionary individual (Doolittle and Booth 2017; Lloyd and Wade 2019). Finally, I present my concluding remarks.

\section{Multispecies individuals}

Defenders of HTE have claimed that holobionts are evolutionary individuals and have presented different trends of evidence to justify their claim. According to Bourrat and Griffiths, their arguments constitute cases of what they call "part of the system" arguments, that would have the following logical structure: "such-and-such components are essential to the functioning of some larger system, therefore those components are part of that system". This line of reasoning, applied to holobionts (system) and their symbionts (components) might be explicated as follows:

(i) Symbionts $x, y, z$ are essential for the functioning of the holobiont $H$

(ii) Therefore, $x, y, z$ are parts of $H$.

Notice that the argument contains an additional elliptical premise that is required to be valid, namely:

(iii) If a symbiont $X$ is essential for the functioning of a holobiont $H$, then $X$ is part of $H$.

Furthermore, Bourrat and Griffiths assume that when defenders of HTE defend (ii), what they are indeed claiming is that:

(iv) Therefore, $H$ is an evolutionary individual.

\footnotetext{
${ }^{10}$ To clarify, by "trait” I do not mean "phenotypic trait", but its the genetic basis (may be e.g. a gene, or a collection of genes in epistatic interaction, etc.). Also, I do not necessarily reduce genes to the DNA content of the genome, but I rather refer to what Lu and Bourrat (2017) have characterized as the "evolutionary gene" (see also Griffiths and Stotz 2013). My reasons for calling them "traits", though, rest on the fact that the studies on the influence of the microbiome on animal evolution I build on refer to this genetic content as "trait", plus the fact that if I used the concept of "gene", I suspect it would be mostly taken to imply solely DNA.
} 
And, importantly, for Bourrat and Griffiths an entity is an evolutionary individual if and only if all the parts of the unit have their fitness interests aligned (unit of selection = fitness alignment). To quote: "the fundamental issue in identifying new levels of biological [evolutionary] individuality should be whether some entity can function as a unit of evolution, which will depend on the fitness alignment between the partners over evolutionary timescales” (Bourrat and Griffiths 2018: 2).

Once the debate has been framed, Bourrat and Griffiths dedicate the rest of the paper to argue that premise (iv) does not follow from (i) and (iii), at least if (i) and (iii) are supported by the kind of evidence that defenders of HTE have used to justify them, simply because (iii) would be false. This is because the arguments use by HTE defenders are not directed to the right kind of properties that would justify considering that a system is a biological individual, but rather to other types of properties which are common to many types of relationships between organisms of different species, and even to relationships between an organism and its abiotic environment (e.g. the gravitational field; Bourrat and Griffiths 2018: 5). As they stand, the authors argue, those properties are not genuine markers of biological individuality. This leads to the second major criticism of Bourrat and Griffiths to HTE defenders: holobiont advocates fail to provide a satisfactory theoretical criterion that allows them to distinguish between holobionts qua evolutionary individuals and other multispecies assemblages qua consortia of interdependent evolutionary individuals. In their view, the real criterion for determining whether a biological consortium is an evolutionary individual is the fitness alignment among the parts that constitute it, an argument that defenders of HTE have not made. Therefore, defenders of HTE have failed in their defence of the notion that holobionts are units of selection, and a new criterium based on the concept of fitness alignment must be made explicit that allows divorcing true multispecies evolutionary individuals from mere multispecies assemblages.

\subsection{Criticism to the part of the system arguments}

Bourrat and Griffiths believe that the arguments presented by defenders of HTE to support (i) are based on (false) premises that make that the deduction of (iv) does not work. In their view, defenders of HTE have used three sources of support for (i): metabolic (physiological) arguments, developmental arguments, and immunological arguments. The metabolic argument, especially emphasized by Dupré and O’Malley (2009), although also present in other formulations of HTE (Gilbert et al. 2012, 2017; Rosenberg and Zilber-Rosenberg 2013, 2016; Roughgarden et at. 2018) basically states that: insofar as macrobes engage in numerous interactions with their symbionts to achieve the basic goal of processing the external resources of their environment to keep the system (host + microbes) alive, the whole system should be considered a biological individual. Furthermore, in the case of Dupré and O'Malley the argument is complemented with the idea that those systems should be considered "the most 
fundamental unit of selection” (2009: 13). To reach their conclusion, Dupré and O’Malley rely on the distinction between interactors and replicators (Hull 1980; Lloyd 2017a), adducing only that holobionts are interactors, and making clear that holobionts are systems composed of independent (sic) "lineage-forming entities".

Bourrat and Griffiths do not have any concern with Dupré and O’Malley’s defence of the claim that holobionts are interactors (cf. Booth 2014; Skillings 2016; Queller and Strassmann 2016). Their only concerns are: Firstly, that if interactors are defined that way, the definition would also be applicable to other multispecies assemblages that are not holobionts; secondly, that "although the notion of an interactor is useful in certain contexts within evolutionary theory (...), it is often of very limited value in deciding whether a multispecies entity is an individual in its own right" (2018: 11, emphasis added). Their first observation is supported by comparing the metabolic system of the worm Olavius algarvensis and its microbial symbionts, with the system formed by the seagrass meadow, bivalves of the Lucinidae family and their endosymbiotic sulphide-oxidizing bacteria, and the sulphate-reducing bacteria that reside next to the roots of the seagrass. Bourrat and Griffiths' point is that although the metabolic processes -including some extremely complex biochemical exchanges- that occur within the $O$. algarvensis and those that occur among the members of the seagrass meadow multispecies assemblage are basically the same, only the former but not the latter would be considered a holobiont by HTE defenders. Then, there would be no theoretical ground to distinguish between holobionts and other multispecies systems, apart from a basic intuition of what seems to be more an individual from our perspective. ${ }^{11}$ And now, and always according to Bourrat and Griffiths, even assuming that holobiont advocates had a convincing argument to show why one system is a holobiont whereas the other is not, they would have only proven that holobionts are interactors. But since the notion of interactor, as Dupré and O’Malley (and others) define it, is of very limited value for deciding whether a multispecies system is an evolutionary individual, ${ }^{12}$ holobiont advocates have totally missed their target, and their evidence is simply not to the point. Therefore, no matter what, HTE advocates fail to provide a satisfactory criterion to support why the metabolic argument leads to the conclusion that holobionts are evolutionary individuals.

\footnotetext{
${ }^{11}$ Similar claims can be found in late $19^{\text {th }}$ and early $20^{\text {th }}$ century history of biology, always directed against those biologists that emphasized the integrative nature of symbiosis. Maybe the most interesting case is Pound (1893), reviewed in Suárez (2018: 80-82).

12 Notice that their observation is not that the concept of interactor is irrelevant in evolutionary theory (cf. Okasha 2006; Godfrey-Smith 2009), but that as the concept is defined by HTE advocates, it is of little utility for deciding whether a multispecies system is an evolutionary individual. I think Bourrat and Griffiths' point to that end is fair, although as I will argue later, they have missed some consequences of the interactor claims as they are formulated by HTE advocates that suggest that there is an evolutionary fact of the matter in arguing that holobionts are evolutionary individuals (section 3, section 4).
} 
The other two arguments that Bourrat and Griffiths discuss (developmental, immunological) have the same conceptual structure, so for reasons of space I will only consider the metabolic argument for the rest of the paper.

\subsection{The criterion of fitness boundedness through fitness alignment}

After the more "destructive” part of their paper, Bourrat and Griffiths formulate an original theoretical proposal that, in their opinion, overcomes the difficulties that the arguments presented by defenders of HTE encounter. In their view, HTE advocates might be after the right sort of intuitions in their defence of the thesis that some multispecies assemblages are evolutionary individuals. But for their intuitions to be correctly captured and able to divorce those that are evolutionary individuals from the rest of multispecies assemblages, HTE arguments must be theoretically grounded on the concept of fitness alignment through fitness boundedness. ${ }^{13}$

Bourrat and Griffiths define fitness alignment as follows:

“(Fitness Alignment) Considering both a spatial and a temporal scale at which an ensemble can be divided into units, the higher the probability that manipulating properties of one unit reliably leads to effects of the same magnitude and direction on the realized fitness of all the partners and only the partners constituting that unit at the temporal scale considered, the more highly each unit scores on individuality” (2018: 16)

Fitness alignment is argued to provide a demarcation mechanism for any group of entities, in the sense of being a "mechanism that increases or maintains the capacity of an object to undergo between-object selection" (Clarke 2013: 424). Second, it is believed to be a criterium for detecting evolutionary individuals: If a group of entities is detected to have its fitness interests aligned, then it might be argued to be fitness bounded, and thus be an evolutionary individual. Third, it is argued not to be an "all or none" criterium, in the sense that it allows different degrees of individuality, depending on how much the fitness of the entities of a consortium are aligned. Finally, it is said to allow accommodating the well-established fact that "conflicts are rampant", in the sense that no group of entities will exist such that there is absolutely zero conflict among the entities in the group (Moran and Sloan 2015: 6; Douglas and Werren 2016; cf. Lloyd and Wade 2019).

\footnotetext{
${ }^{13}$ This criterion may be interpreted as a formulation of the conditions for being an interactor for multispecies assemblages. Thanks to Pierrick Bourrat for clarifying this point to me.
} 
With the fitness alignment criterium in mind, Bourrat and Griffiths proceed to argue that some of the original intuitions of HTE advocates about the difference between the evolutionary individuality of holobionts and the evolutionary individuality of other multispecies assemblages can be explicated: In the case of some holobionts $-O$. algarnvensis system- the fitness alignment that ties the partners together is higher than the fitness alignment that ties the partners together in the case of other multispecies assemblages -seagrass meadow system-. Concretely, they argue that the entities that constitute evolutionary multispecies assemblages (and, in some cases, holobionts) have their fitness interests bounded, so that "[m]anipulating a character of a unit would certainly affect the fitness of all the partners of a unit with the same or a very similar magnitude and in the same direction” (2018: 17, emphasis added). The situation, however, is not the same for the seagrass meadow system, which "seems to offer no reason to believe that a manipulation would, in general, lead to a similar magnitude and direction of fitness effect on each partner" (2018: 18, emphasis added).

Therefore, for Bourrat and Griffiths, the fitness alignment criterium would solve the main issues that they find with the arguments presented by HTE defenders, as it allows to clearly distinguish the symbiotic associations that give rise to evolutionary individuals from mere multispecies assemblages that cannot be considered as such. But, at the same time, it has a very important consequence for HTE, as it denies that holobionts (host-microbiome associations) have any privileged status as symbiotic evolutionary individuals that could not be predicated of any other type of symbiotic assemblage. However, as I will argue, Bourrat and Griffiths have not paid enough attention to a possibility (stability of traits) that would render most holobionts evolutionary individuals in their own right, and thus as special units at which the part of the system arguments must be primarily addressed. I will elaborate that argument in the next sections.

\section{The evolutionary basis of the part of the system arguments: The case of the vampire bat}

In this section, I draw on the case of the hologenomic adaptations that underlie the evolution of sanguivory (hematophagy) in vampire bats (Desmodus rotundus) to justify the evolutionary implications of the so-called "part of the system" arguments invoked by HTE defenders (Mendoza et al. 2018). The key claim in this section will be that although part of the system arguments are not directly evolutionary, they are so indirectly, as they are used as approximations to suggest that part of the current biological features of hosts are the result of a selection process in their microbiome. Importantly, I argue that this selection process does not necessarily affect the lineages 
that make up the host microbiome -although that could happen in some cases-, but the traits (genes, genetic components that code for a biological function) that are encoded in it. In that vein, I argue that part of the system arguments are indicative of the collection of microbiome traits that, together with the host genome, constitute the evolutionary individual that we call "hologenome".

Bats (order Chiroptera) are the second largest order of mammals and are the only ones whose forelimbs have evolved as wings, thus developing the capacity to fly. Due to the large number of species included in the order, it includes several species and a wide variety of nutritional adaptations. Most bats are frugivores (fruit-eaters), but some of them are also insectivorous (insect-eaters), and yet some are sanguivorous (or hematophagous, blood-eaters). About this last point it is important to note that obligate sanguivory in mammals is exclusive to three bat species (clustered together in the family Phyllostomidae Desmoodontinae), and it has not evolved as such in any other mammalian order. This fact suggests that the evolution of sanguivory must have followed a unique evolutionary trajectory in the order Chiroptera that is not present in any other mammal.

Importantly, obligate blood-sucking diet is a challenging lifestyle which generates a series of systemic biological challenges that are not evolutionarily easy to cope with for any system. First, and foremost, blood is not very nutritious: it basically consists of proteins (93\% in its dry-matter phase), and its content of fats, carbohydrates and vitamins is really scarce. Its extremely high levels of protein content may easily lead to kidney and bladder failure. Additionally, its high levels of iron are disruptive of liver, heart, and pancreatic function. Second, blood is full of pathogens, many of which are exclusive to it (bloodborne pathogens), and extremely harmful if they spread to any other bodily part. Third, blood tends to coagulate when it is not circulating under highly specific conditions. Vampire bats need to produce anticoagulants in their saliva to avoid blood coagulation. Fourth, blood is not an easy nutritional source to locate, requiring highly specific morphological and sensory-motor adaptations (Figure 1). On the one hand, they need very sharp canines and incisor teeth specifically evolved to pierce the blood vessels of their prey and suck their blood. On the other hand, they need specific sensory organs to detect the blood vessels of their prey, as well as claw-thumbed wings to adhere to their prey while they feed on it. 

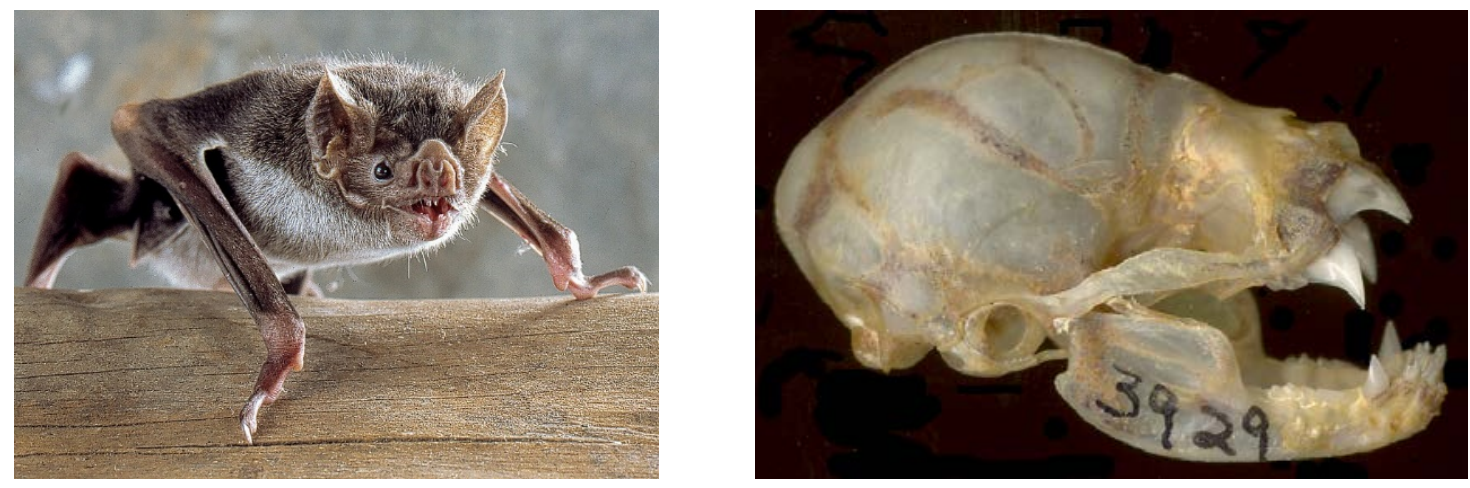

Figure 1. Image of a common vampire bat (left), and of a common vampire bat skull (right). The morphological adaptations to sanguivory can be clearly appreciated in the image on the left. The wings are claw-thumbed, to avoid its prey from scaping, and the head has adapted for the development of sucking teeth. The nose has a heat sensor that allows detecting blood vessels, and two incisive teeth can be appreciated. The image on the right shows the teeth profile of vampire bats. Incisive and canine teeth are salient, and it may be observed how vampire bats have considerably reduced the size of their molars, which have almost completely disappeared. Images from Wikipedia Commons., https://en.wikipedia.org/wiki/Common_vampire_bat\#/media/File:Desmoboden_(cropped).jpg ;

https://en.wikipedia.org/wiki/Common_vampire_bat\#/media/File:Common_Vampi re_Bat_Skull.jpg. Accessed on the $12^{\text {th }}$ of January 2020.

Two key questions for biologists working on vampire bats concerns first, how vampire bats can cope with the challenges posed by sanguivory; second, how the family Phyllostomidae Desmoodontinae has evolved so that they are now the sole mammal order with an obligate blood-sucking diet. In other words, they need to investigate to find, at least, a functional and a historical explanation of how vampire bats are the type of creatures they are now, and how they have evolved to become the type of organisms that they are. Notice that the two questions may be related, for what vampire bats do now is, at least in part, a result of their evolutionary history. Thus, biologists need to understand both how vampire bats cope with the selective pressures derived from their hematophagous diet as a previous step to investigate where to locate the traits that have been naturally selected to cope with these environmental challenges.

Following these two questions a recent study by Mendoza et al. (2018) has demonstrated that the evolution of sanguivory in bats is not the result of a selective history in the bat genome alone. Although changes in the bat genome explain key changes that arguably lead to the evolution of sanguivory and prove that the members of the family Phyllostomidae Desmoodontinae followed a different evolutionary trajectory than other families of bats, they fall short of showing how vampire bats can cope with the challenges posed by hematophagy. Especially, how vampire bats cope with many of 
the nutritional and immunological challenges posed by sanguivory. To cover that gap, Mendoza et al. decided to apply an hologenomic approach, and rather than only focusing on the genomic changes that may have led to the evolution of hematophagy, they additionally studied the changes in the genetic composition of the microbiome. Building on that approach, their results were clear: many of the traits that have evolved to allow the evolution of sanguivory in the family Phyllostomidae Desmoodontinae are microbiome adaptations, rather than genomic adaptations (Figure 2). Concretely, several traits of the microbiome that are under specific selection in vampire bats are there as a consequence of their functional role in coping with the nutritional and immunological challenges of sanguivory, suggesting the existence of a selective history for those traits at the hologenomic level, rather than at the level of each of the bacterial lineages.

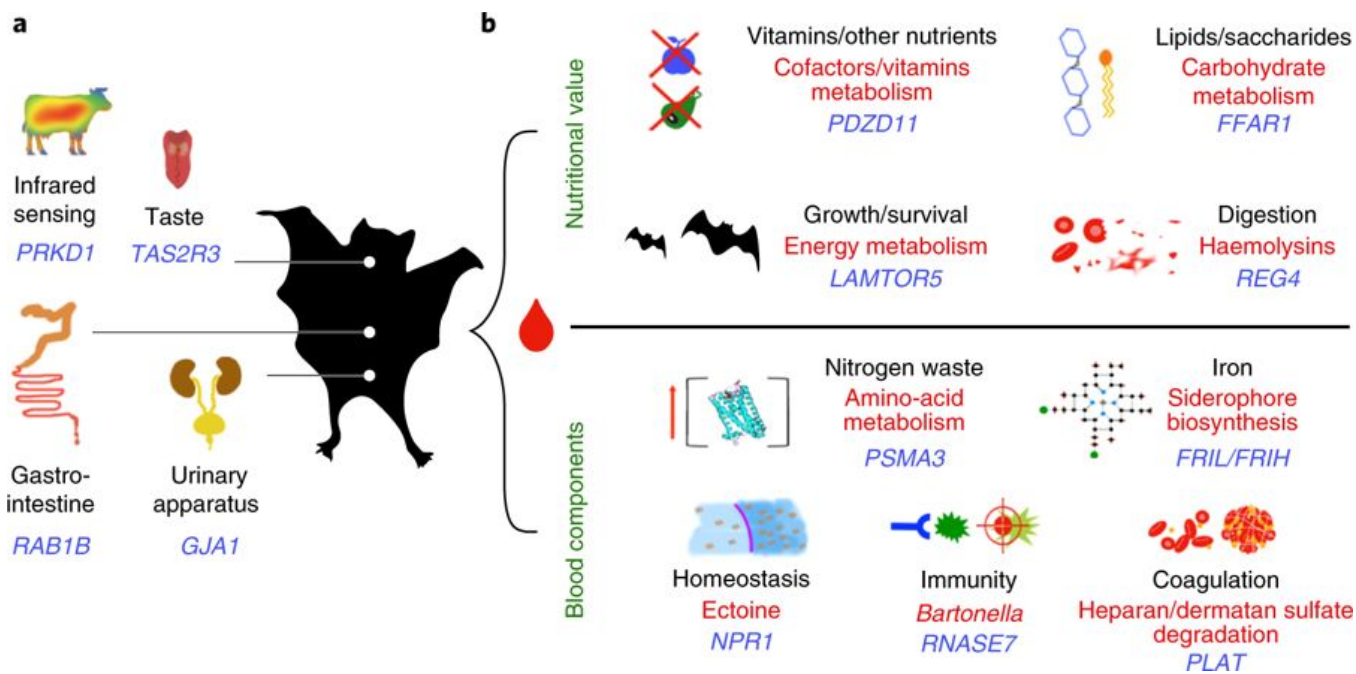

Figure 2. Type of adaptations in the vampire bat, and specific contributions of the bat genome and on the bat microbiome to cope with the challenges of sanguivory. On the left, traits that have evolved exclusively in the bat genome. On the right, adaptations to cope with sanguivory that evolved both in the bath genome (blue), and in the microbiome (red). The figure shows how most microbiome adaptations are allow bats to cope mainly with nutritional challenges, although also with other physiological challenges such as immunity. From Mendoza et al. 2018: Fig. 2. Copyright: Creative Commons. Reproduced with permission.

Let me explain the last point more clearly, as well as why the importance of the hologenomic framework is especially salient in this case. In their research, Mendoza et al. found positive selection for traits in the bat genome whose evolution can be clearly associated to their dietary requirements. To list a few, they demonstrated that genes RAB1B, GJA1, PSMA3, REG4, PLAT, PDZD11, LAMTOR5, FFAR1, or RNASE7, among others, showed a history of positive selection in common vampire bats that suggested that they were adaptations specifically evolved to address the selective 
pressures of blood-eating. For instance, $R A B 1 B, G J A 1$ and PSMA3 very likely evolved in the family Phyllostomidae Desmoodontinae to cope with the potential kidney and bladder problems derived from the risks of nitrogen degradation that usually appear in cases of blood consumption. PLAT and REG4 genes are believed to be involved in anticoagulation, including the production of anticoagulants in the saliva. Also, RNASE7 is known to be an antimicrobial gene, and in the case of vampire bats in can be consistently correlated with their necessity to constantly cope with bloodborne pathogens.

As I said, all of these genomic adaptations alone are insufficient to explain the evolution of sanguivory. An analysis of the microbiome is also required, and this was thus the next step in Mendoza et al.'s work. This was carried out in two steps: first, a taxonomic analysis of the bacterial lineages that compose the bat's microbiome; second, a functional analysis of the genetic components -irrespectively of the taxa they appear on- that are present there. The taxonomic analysis revealed little differences between the vampire bat microbiome and the microbiomes of frugivorous and insectivorous bats. Their hypothesis was that the differences between the vampire bat's microbiome and the microbiomes of other bat families could be explained taxonomically, as simply reflecting the phylogenetic differences between the different bat families (i.e. suggesting a pattern of phylosymbiosis) (Mendoza et al. 2018: Fig. 3). However, as the microbiome taxonomic differences between bat families were so little, it was hard to understand how the vampire bat microbiome could play any role in coping with the challenges of sanguivory in any significant sense. To cover that gap. Mendoza et al. studied the functional profile of each of these microbiomes. In contrast with the taxonomic analysis, the functional analysis provides biochemical information about the genes that are present in the microbiome, and thus generate different classifications and data-sets than taxonomic classifications (Suárez 2016; Lemanceau et al. 2017). Their observation was surprising for they observed that "the vampire bat microbiome is strikingly different to that of the compared bats at the functional level (...). While there is little differentiation between the functional gut microbiomes of carnivorous, insectivores and frugivorous bats, the common vampire bat functional gut microbiome is almost completely distinct, and exhibits the least intra-species variation between the samples.” (Mendoza et al. 2018: 661). In fact, the high degree of functional dissimilarity between the vampire bat microbiome and the microbiome of other bat species strongly suggested that the vampire bat microbiome contained a number of traits directly involved in the challenges of sanguivory that clearly outnumbered the number of genes that played the same roles in the vampire bat genome (Figure 3). 

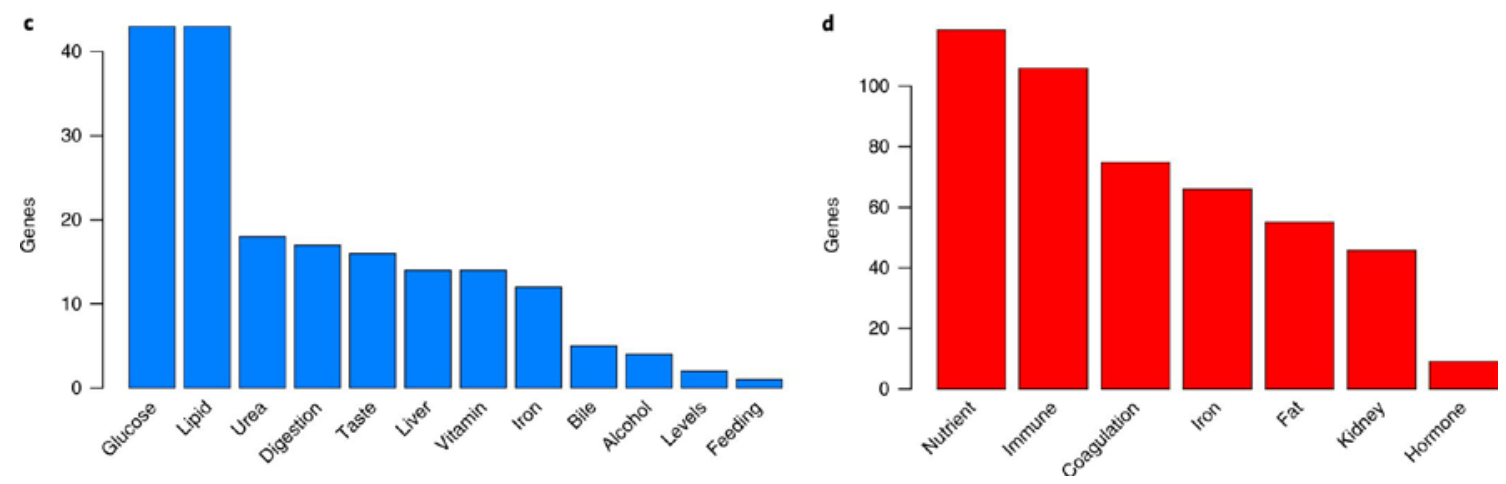

Figure 3. Number of traits in the host genome that have evolved in direct relation to sanguivory (left), versus number of traits in the microbiome that have done so (right), as revealed by Mendoza et al. (2018). From Mendoza et al. 2018: Fig. 4. Copyright: Creative Commons. Reproduced with permission.

These results show that the microbiome, far from being selectively neutral, as Bourrat and Griffiths argue, is of primary selective importance in the evolution of some animal families. In the case of vampire bats, microbiome evolution is probably the best way to explain why vampire bats' genomes are so "unprepared" to cope with the challenges of sanguivory, despite being obligate blood-eaters. The evolution of vampire bats has only been possible because, in addition to the evolution of the bat genome, the microbiome has functionally evolved in a specific way that allow the members of the family Phyllostomidae Desmoodontinae to cope with the challenges of sanguivory. In Mendoza et al.’s view:

"It is clear from our results that the common vampire bat has adapted to sanguivory through a close relationship between its genome and gut microbiome. (...) We showed that extreme dietary specializations, such as that of the common vampire bat, provide a comparative framework with which to tease apart the relative roles of genomes and microbiomes in adaptation. In conclusion, our study illustrates the benefits of studying the evolution of complex adaptations under $a$ [sic] holobiome framework, and suggests that vertebrate adaptation studies that do not account for the action of the hologenome may fail to recover the full complexity of adaptation” (Mendoza et al. 2018: 664, emphasis added).

Concerning the so-called "part of the system” arguments, Mendoza et al.'s research suggests that, even while these arguments are normally not formulated in evolutionary terms (as Bourrat and Griffiths fairly point out), they do indeed mask an evolutionary story of why some hosts (animals, plants) have the biological features they do. Concretely, they mask a story of how the microbiome and the host genome have evolved so that contemporary animals and plants can cope with the selective pressures 
they must cope with nowadays, including niche adaptations or dietary adaptations. Thus, far from being evolutionary trivial, part of the systems arguments are provisional arguments about the possible type of adaptations that the microbiome may have gone through, given the current physiology, development or immunology of the animal or plant species whose evolution is being studied. And these evolutionary changes, while fundamental for the host survival, do not need to result from the co-evolution or cospeciation of the host and the species that compose its microbiome (Brooks et al. 2016; Theis et al. 2016).

Drawing on this, it seems now justified to argue that Bourrat and Griffiths have not paid enough attention to an alternative interpretation of premise (i), as well as the elliptical premise (iii) in the so-called "part of the system" arguments. They interpret that the units whose fitness interests are bound are the host lineage and the lineages that compose the microbiome. Their interpretation, indeed, is fair to what defenders of HTE have sometimes argued, according to which the hologenome is the collection of genomes of all the entities that compose the holobiont (Zilber-Rosenberg and Rosenberg 2008; Rosenberg and Zilber-Rosenberg 2013; Bordenstein and Theis 2015; Theis et al. 2016; see section 4.1). However, I claim that they have ignored a possibility that the case of vampire bats illustrates, namely: that the units whose fitness interests are bound are the host genome and the traits that compose the host's microbiome. As this possibility is ignored, Bourrat and Griffiths reasonably come to believe that the deduction from (i) to (ii) -and thus to (iv) - is unsound, for the premises are not to the point (i.e. they do not say anything about the evolution of the holobiont, thus they cannot be used to argue that holobionts are evolutionary individuals). However, this is not the case if these arguments are understood as suggestions that some traits encoded in the microbiome are evolving as part of an extended host genome, or hologenome. I will elaborate what that view entails more succinctly in the next section.

\section{Back to part of the system arguments: The stability of traits account of holobiont evolutionary individuality}

Although the case of the common vampire bat strongly suggests that hologenome selection, and thus HTE, is an empirical reality (and thus holobionts are evolutionary individuals), it still requires some interpretation to overcome the difficulties raised by Bourrat and Griffiths. To recall, their argument was that a real criterion for evolutionary individuality was the concept of fitness boundedness through fitness alignment. Thus, their argument was that the movement from premise (i) to (ii), and from these to (iv), required of specific mention to the fitness interests of the partners that associate symbiotically. Otherwise, defenders of HTE fail to provide a consistent evolutionary claim that tells real multispecies evolutionary individuals apart from other types of multispecies assemblages. In this section, I will make a stronger claim that the one I 
made before, for I will argue that "part of the system" argument do indeed, in certain cases, mask claims about the fitness boundedness of the entities that compose a holobiont. Concretely, I argue first that the nature of the microbiome is such that it may be considered as a functional unit, for many of the traits within it are not necessarily linked to a specific bacterial lineage; second, that the entities that have their fitness interests aligned are the host genome and the functional traits of its microbiome, a possibility that Bourrat and Griffiths ignore. These two claims lead me to propose a reformulation of the concept of the hologenome in the HTE framework. I claim that the evolutionary individual does not consist in the sum of genomes that make up the hologenome, but in the sum of the host genome plus certain traits of its microbiome. This leads to a reformulation of the part of the system arguments as:

(i') Traits $a, b, c$ are essential for the functioning of the holobiont $H$

(ii') Therefore, $a, b, c$ are parts of $H$

Let me start this section with a quote from a very influential paper in the hologenome literature by Theis and colleagues:

"Microbial genomes can be stable or labile components of the hologenome and can be vertically or horizontally transmitted, and the traits that they encode are context dependent and may result in damage, benefit, or no consequence to the holobiont” (Theis et al. 2016: 2, emphasis added).

From this quote, it seems clear that HTE advocates consider, first, that the hologenome is the entity composed by a host (host genome) plus the microbial genomes that compose its microbiome; second, that those microbial genomes are considered to be of evolutionary relevance for the holobiont in virtue of the traits that they encode. These two claims, although related and treated as a unique claim by hologenome defenders, need to be conceptually divorced if one aims to have a clearer picture of the evolutionary role of host-microbiome multispecies assemblages. On the one hand, as Bourrat and Griffiths argue, not all the genomes that compose a holobiont need to be evolutionary linked, and thus it is not necessary that their symbiosis has evolutionary consequences for every genome in the system. But, on the other hand, as the case of the vampire bat suggests the part of the system arguments may mask some evolutionary facts that involve some of the traits that are encoded in the microbiome, rather than the lineages or genomes that compose the microbiome. These traits will be borne by the bacterial lineages that compose the host microbiome but will be encoded by specific regions of the bacterial genome, and this has important consequences for defending a refined version of the part of the system arguments, as I will develop more clearly in the 
next paragraphs. The question we must now raise is, thus, how to make these two points compatible without equating HTE to a thesis that involves both the host genome and the totality of the genomes that compose its microbiome, or reducing it to a thesis that concerns exclusively the host and the lineages of the microbiome that are vertically transmitted and thus necessarily linked by the criterion of fitness boundedness. The first option, defended by HTE defenders, seems to be empirically false for most systems and, as Bourrat and Griffiths argue, does not allow to divorce true multispecies evolutionary individuals from mere multispecies assemblages. The second, adopted by Bourrat and Griffiths, had however the risk of ignoring cases such as the vampire bat, where there seems to be an evolutionary fact of the matter despite the possible lack of fitness alignment between the genomes that compose the holobiont (Figure 4).
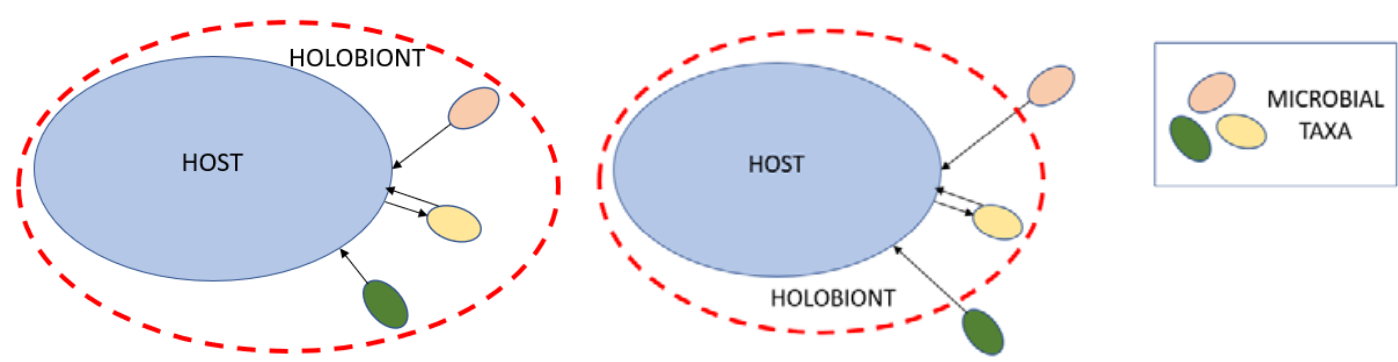

Figure 4. Schematic representations of the boundaries of the holobiont (dashed red ellipse) according to HTE advocates (left), and Bourrat and Griffiths (right). Different colours represent different lineages/taxa. The arrows stand for fitness interactions. A double arrow indicates that the host and the bacterial taxon have their fitness interest bound, such that the death of one will trigger the same effects on the other. The single arrow stands for cases where host fitness is affected by its interaction with the bacterial taxon, but not the other way around. From Suárez (2019: 196, Fig. 25).

Let me illustrate with another example why it is biologically important to consider the contributions of the microbiome to host evolution. In section 1, I explained how some observations about the biology of corals led Rosenberg and Zilber-Rosenberg to the formulation of HTE (see also Lamm 2018). Originally, the hypothesis was directed towards explaining how the different taxa of microorganisms that integrated the microbiota of the host could be transmitted, so that natural selection could act on holobionts, as a unit, to produce cumulative selection. ${ }^{14}$ Therefore variation was

\footnotetext{
${ }^{14}$ It is important to bear in mind that natural selection, to produce cumulative effects and, thus adaptation requires the existence of a population of entities where: (i) different entities exhibit different phenotypic variants, (ii) different variants have different fitness, and (iii) those variants are transmitted (Okasha 2006; Godfrey-Smith 2009). If one of the criteria (i)-(iii) is missing, then the entities might be argued to evolve, but not to evolve by natural selection. Notice that no mention is made to the nature of the entities (i.e. how they must be individuated), nor to the mechanisms by which the variants are transmitted, or the level at which transmission occurs.
} 
conceptually associated with "variation in microbiome composition in taxa". However, some discoveries soon lead to the realization that taxa composition of the microbiome was not as transmittable across host-generations as had been originally thought. And also, at the same time it was observed that taxa composition of the microbiome by itself was sometimes irrelevant for determining the holobiont's phenotype. For instance, Hester and colleagues (2016) observed that coral's microbiome composition in taxa might shift quite dramatically depending on changes in the environmental pressures, while the functional profiles -or the traits encoded by the microbiome- remained more stable. A similar observation on cows was made by Taxis and colleges (2015), who observed high taxonomic variability despite similar metabolic necessities, which suggested the existence of a common core of functional profiles (see also Louca et al. 2016).

Drawing on these observations, and the case of Mendoza et al. (2018), I want to put forward a new conception about the boundaries of the hologenome that I call “stability of traits” conception (see also Suárez and Triviño 2019; Suárez 2019). ${ }^{15}$ It asserts that the evolutionary individual is the entity composed by the host genome and some traits of the microbiome: (1) specifically evolved to cope with the dietary, environmentally, immunological, etc. challenges faced by the host, (2) whose evolutionary significance is contextual, i.e. they can only (or mostly) play the role they play when they are in the host microbiome. As a consequence of this: (a) these traits replace some traits in the host genome, which do not need to be acquired, for the same role is played by an external resource (i.e. by the microbiome); (b) these traits have their fitness interest aligned with the fitness interests of the host, for they can only "survive" if the lineages that bear them are in the host microbiome (Figure 5).
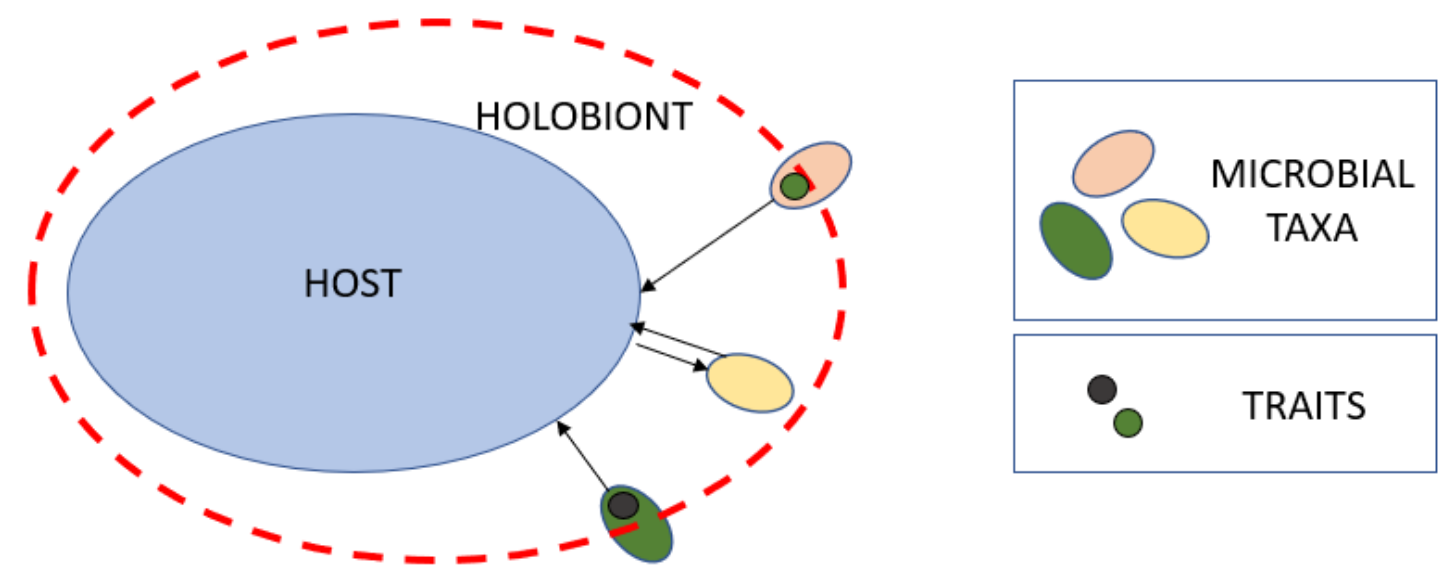

\footnotetext{
${ }^{15}$ A very similar idea applied to the notion of shared history among symbiont species was already suggested in the $19^{\text {th }}$ century by Schneider (1897). See Suárez (2018: 82-84) for a review of Schneider's ideas. Additionally, the account bears some similarity with Bouchard's ideas about fitness and natural selection, although it also constitutes an elaboration of these ideas (Bouchard 2013, 2014).
} 
Figure 5. Schematic depiction of the boundaries of the holobiont according to the stability of traits account (dashed red ellipse). Different colours represent different lineages/taxa, or different traits. The arrows represent the situations where there is some fitness boundedness. The double arrows represent that either the death of the host, or the death of the microbial taxon, will trigger the same effect on both units. The single arrow, however, depicts the situation where the effects will only affect the traits that the taxa that compose the microbiome bear, but not to the taxa. From Suárez (2019: 198, Fig. 26).

Let me illustrate the stability of traits account of the holobiont by drawing on Mendoza et al.'s research. As said in section 3, their research proves that microbiomes that are not very distant from a taxonomic point of view (vampire, frugivorous, insectivorous bats), can be functionally strikingly different, as well as adapted to cope with extremely different dietary conditions. This evidence suggests that while the microbial taxa that compose the host microbiome may be evolutionarily independent from the host (i.e. following Bourrat and Griffiths' criterium, their evolutionary interests are not linked to the host interests and they can survive independently of it), the same is definitely not true for the host genome, and I hypothesize it is not true either for the traits in the microbiome directly evolved to cope with the extreme dietary conditions of vampire bats. ${ }^{16}$ In other words, the hypothesis I am defending is that the vampire bat (and thus, its genome, which is what is relevant at this point) will "die" if it loses its microbiome, and the thousands of traits in the host microbiome directly evolved to cope with sanguivory will also "die" if the bacterial lineages that bear them migrate to a different environmental niche, including another host that does not have a sanguivory diet (e.g. a frugivorous bat). Therefore, the host genome and the collection of traits of the microbiome satisfy the criterion of fitness boundedness through fitness alignment for "the higher the probability that manipulating properties of one unit reliably leads to effects of the same magnitude and direction on the realized fitness of all the partners and only the partners constituting that unit at the temporal scale considered" (Bourrat and Griffiths 2018: 16).

\footnotetext{
${ }^{16}$ Pierrick Bourrat has told me that this would at most prove that microbiome traits are transgenerationally recurrent, but not necessarily that evolution by natural selection is happening at the holobiont level, since for that you also need some form of parent-offspring relationship (or I would be conflating reproduction of with reproduction by, see Ariew and Lewontin 2004). Although I agree with him that mere recurrence would not be enough, but a causal basis for this recurrence is also required, I am not sure that the case I am building on is one of "mere recurrence". First, the hypothesis is that these traits only re-occur on a very restricted population of bats, which even if it does not restrict the recurrence to parent-offspring, it substantially restricts the individuals where this recurrence is possible. Second, because I suspect (although this is open to empirical research) that the reason for this recurrence is causal: it is highly related to the biological nature of vampire bats. It would be necessary to study further the exact causal basis of that recurrence, but the case study strongly suggests that there must be a selective basis behind it.
} 
One may wonder about the reasons to argue that some of the traits of the vampire bat microbiome have their fitness bound to the host genome, rather than to the bacterial lineages that bear them, as this statement needs an argument to support it. Of course, such hypothesis needs empirical support for each system, as well as for each family of traits, but our current evidence strongly suggests that it may be the case. ${ }^{17}$ Firstly, the microbial traits that evolved to cope with the challenges of sanguivory are tightly connected to that type of diet. They include genes such as L-asparaginase, involved in anticoagulation; glycerol kinase, involved in the synthesis of triacyglycerol; or ureA, involved in urea degradation and thus highly related to kidney function. Many of these genes are either exclusive to the vampire bat microbiome, or highly enriched in it, suggesting that they are directly related to hematophagy, rather than directly involved in the survival of the bacterial lineages that bear them. This strongly suggest that any of these traits will be inefficient in any other niche, including in other bat families, and thus they will either be eliminated by natural selection (for they would reduce the fitness of the bacterial lineages that bear them) or their content would be substantially reduced. Thus, these functional traits seem to be in the vampire bat microbiome and only in the vampire bat microbiome, possibly due to some specific form of epistatic interaction between the host and its microbiome (Lloyd and Wade 2019; Bourrat 2019). No matter how, this evidence suggests that the fitness of these traits is completely bound to the fitness of their host. ${ }^{18}$

Secondly, the same does not hold true for the bacterial lineages that compose the bat microbiome. Furthermore, it is not expected to hold true in most evolutionary scenarios neither from the host perspective, nor from the perspective of the microorganisms that bear the traits. On the one hand, fitness boundedness between different lineages is known to be rare, and it is known to give rise to dangerous evolutionary scenarios for the host lineages that engage in that type of patterns, creating the possibility of what has been called an evolutionary "rabbit hole". That situation occurs when the two lineages evolve such strong dependencies that led to the degeneration of symbiont genomes. In that scenario, the ecological niche of the host species is ultimately reduced, which may lead to its extinction (Bennet and Moran 2015). On the other hand, the bacterial lineages that compose the host microbiome will benefit very little from an obligate interaction with the host, as it will reduce the potential niches where they can survive. The best scenario for the lineage that compose the microbiome is to have the capacity to acquire/lose motile elements that are directly connected to the host lifestyle. In that vein, they can acquire the traits when they are

\footnotetext{
${ }^{17}$ I will only discuss the case of the microbiome for it is generally accepted among scientists working on bat biology that the vampire bat genome, alone, is clearly insufficient to keep vampire bats alive on the basis of a blood-sucking diet (Mendoza et al. 2018).

${ }^{18}$ Of course, one may argue that the lineages that bear the traits would still keep them if their new niche is another species of vampire bat, for the family Phyllostomidae Desmoodontinae includes three species. That is true but notice that this does not invalidate the claim that the microbiome traits have their fitness interests aligned to a very reduced set of host genomes.
} 
host symbionts and lose them when they live in a free environment, so that they are better adapted to their specific selective pressures (Stencel 2016; Stencel, forthcoming).

Finally, the generalized existence of functional redundancy in the microbiome of most macrobes (Boon et al. 2014; Vieira-Silva et al 2016), together with the observation that horizontal gene transfer (HGT) among microorganisms is higher among those that compose a host's microbiome than among their free-living counterparts (Liu et al. 2012; Lerner et al. 2017), reinforces the stability of traits account. First, as the life cycles of macrobe hosts are longer than the life cycles of the components of their microbiome, opportunity for selection in the microbiome is high. This may likely increase the availability of these traits directly related to the host life style, and that are not directly available in its genome, thus increasing their level of redundancy (Díaz 2015). Second, as HGT is substantially increased in the microbiome, it being "a hotspot for horizontal gene transfer" (Stephanie Schnorr, personal communication), it is expected that these traits get passed between different bacterial lineages (Figure 6). In fact, it is expected that the host will tend to favour this, as this is a way of guaranteeing a higher availability of these traits for future generations. Furthermore, this is also beneficial for the bacterial lineages in the long-term, insofar as the capability of acquiring new traits (and getting rid of useless traits) increase their probability of adapting to rapidly changing niches. Finally, and not less importantly, it is beneficial for the traits themselves, as their only way of guaranteeing their persistence is by ensuring that the bacterial lineages that bear them symbiotically interact with the host where they become useful. Therefore, it is expected that during the host life cycle the traits directly related to the host lifestyle increase their frequency in the microbiome and increase their presence in different bacterial lineages. ${ }^{19}$

\footnotetext{
${ }^{19}$ Notice that the model I present here is strictly speaking only an argument to consider holobionts as evolutionary individuals from a multilevel selection 1 or interactor perspective (Okasha 2006; Lloyd 2017a). However, it differs from other similar approaches (e.g. Roughgarden et al. 2018; Roughgarden 2019) because it emphasises the possibility that the microbiome traits that get their frequencies increased do so in virtue of being shared among different bacterial lineages, rather that in virtue of being increased within the bacterial lineage where they originally appeared. This thus opens the possibility for a new way of conceiving the possible role of the holobiont from a multilevel selection 2 perspective, i.e. as a reproducer.
} 

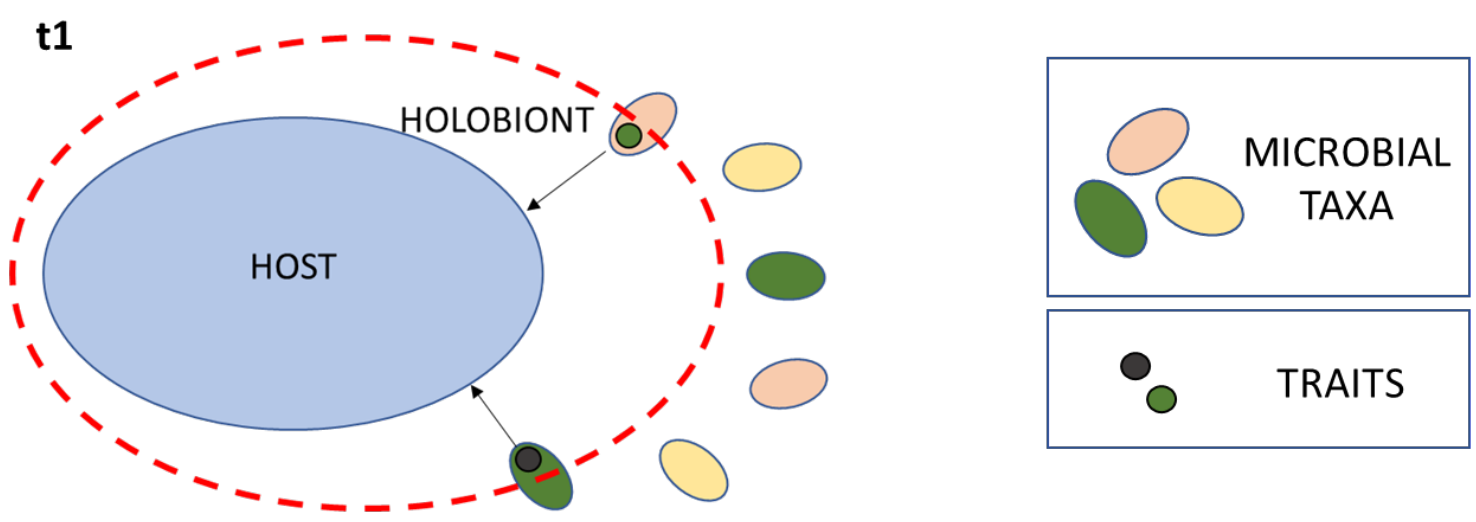

t2

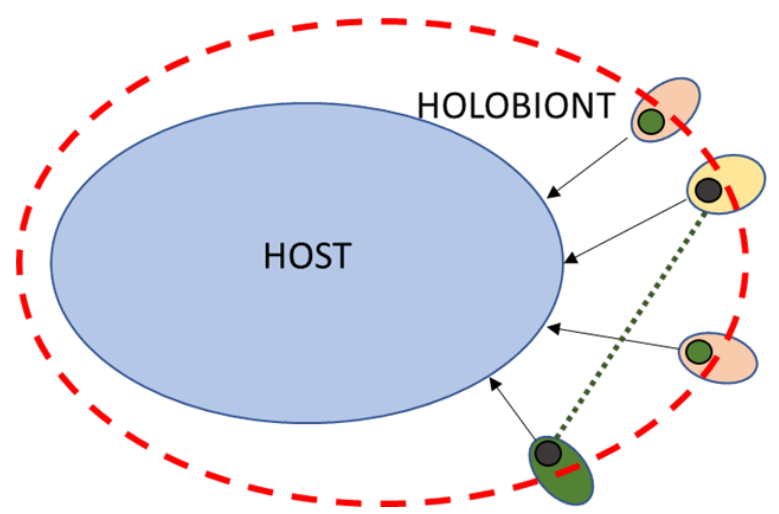

Figure 6. Schematic representation of the ontogeny of the holobiont (dashed red ellipse). $t 1$ and $t 2$ represent two different temporal stages in host development. The arrows stand for cases of fitness alignment where the lineages are not aligned, but only the host genome and some of the traits in the microbiome are. Different colours represent different lineages/taxa, or different traits. The dotted green line represents an event of horizontal gene transfer between bacterial lineages. As the host persists longer than the microorganisms of the microbiome, one possible outcome is that the traits that together with the host genome constitute the evolutionary individual according to the stability of traits hypothesis get amplified and become functionally redundant. In the most extreme case, these traits may even get passed to different bacterial taxa, so that they can increases their probability of surviving across different host generations as it occurs between the yellow and the green taxa. Adapted from Suárez (2019).

This said, there is now a way to show how it is possible to see why the part of the system arguments not only mask an evolutionary fact of the matter (section 3), but even suggest that the holobiont is an evolutionary individual, at last according to the stability of traits account of the hologenome. For part of the system arguments, either in their metabolic, developmental, or immunological form suggest, first, that there are certain functions that the host genome cannot carry out alone, and for which specific traits must have evolved; second, that at least some of these traits have evolved in the microbiome; third, that some of these traits can only exist in the microbiome of very few host 
lineages (sometimes even one or two species), and would probably not exist otherwise, as their fitness interests are aligned to the fitness interest of the host genome, rather than to the fitness of the bacterial lineages that bear them.

Refined in this way, the part of the system arguments are in fact to the point, as they suggest which traits must be looked for, and where (in the microbiome), in order to adequately uncover the evolutionary history of many macrobe lineages. ${ }^{20}$ In addition to that, this refinement of the part of the system arguments suggest why those traits must be considered part of the hologenome as an evolved unit, rather than part of the bacterial lineages that bear them. For, as I argued, these traits have their fitness interests aligned with the host genome, rather than with the genome of the microorganisms that bear those traits. ${ }^{21}$ Finally, this refinement of the part of the system arguments points to a strong asymmetry between claims about the microbiome and claims about other environmental factors that may influence host metabolism, development or immunology -Bourrat and Griffiths give the example of the gravitational field. Concretely, they show that some of the collection of genes that compose the microbiome are evolving because they compose the holobiont, and in doing so they evolve differently to how they would do if they did not. And this includes the host genome too. Notice that the same cannot be said about the gravitational field, which would be the same irrespectively of whether there are organisms whose metabolism, development, or immunology it can affect (see also Stencel and Proszewska 2017; Suárez and Triviño 2019; Lloyd and Wade 2019; Suárez and Stencel, under review).

If the stability of traits account of the evolutionary holobiont holds true for some systems (such as vampire bats), then the hypothesis would give rise to some predictions that are to be expected in these systems, at least in the cases where there has been a strong degree of hologenomic selection:

- First, that the functional redundancy in the microbiome will mostly affect to the traits directly related to cope with the selective pressures faced by the host.

\footnotetext{
${ }^{20}$ The strength of hologenomic selection will of course depend on the strength of selection at the level of the lineages that compose the host microbiome, as well as on the possibility of the specific traits undergoing hologenomic selection to get transferred horizontally to different lineages in the microbiome. This fact can may hologenomic selection inefficient in some cases. However, whether it is inefficient or not is open to empirical investigation and cannot be solved merely on a conceptual basis.

${ }^{21}$ Importantly, nothing of what I have said entails that the traits in the microbiome are coevolving with the host genome. This may be the case, and that situation would create more pressure for these traits to become motile elements. However, it is not necessarily so. An evolutionary individual is a unit whose elements have their fitness interests aligned, but not necessarily a unit whose elements are coevolving. See Brucker and Bordenstein $(2013,2014)$ for an excellent argument to that end.
} 
- Second, that the traits of the microbiome that are directly evolved to cope with the challenges of the host will be involved in more events of HGT, and will probably be present in several bacterial lineages in the microbiome.

- Third, that the part of the microbiome that will be transgenerationally preserved will be its functional core, rather than its taxonomic content, and thus the possible role of the holobiont as a reproducer needs to be thought of from that perspective.

\subsection{The stability of traits account of the holobiont and other accounts of the holobiont}

The account of the holobiont I have just presented is ontologically different from most accounts that claim that the holobiont is an evolutionary individual (or a unit of selection). For space reasons, I will only discuss these differences briefly and in relation to three accounts.

- Hologenome theorists. Most defenders of HTE assume that the evolutionary individual (or hologenome) is the collection of genomes that compose the holobiont (Zilber-Rosenberg and Rosenberg 2008; Rosenberg and ZilberRosenberg 2013; Bordenstein and Theis 2015; Theis et al. 2016, Roughgarden et al. 2018). But, as many critics of the holobiont have argued, including Bourrat and Griffiths (2018), the collection of genomes lack fitness alignment and thus natural selection from below will always disrupt natural selection from above (Moran and Sloan 2015; Douglas and Werren 2016; Stencel and Wloch-Salamon 2018). The stability of traits account, by putting the attention on the traits that compose the microbiome, rather than on the lineages, overcomes that difficulty. The evolutionary individual is the entity composed by the host genome and some of the traits if its microbiome, whose discovery is open to empirical investigation.

- $\quad$ The song, not the singer account. Doolittle and his collaborators have recently suggested the existence of a new type of evolutionary individual that would be individuated for the type of processes it carries out ("patterns of interaction”), rather than from the entities that carry these processes out (Doolittle 2017; Doolittle and Booth 2017; Doolittle and Inkpen 2018). In their view, processes such as geochemical cycles, or the nitrogen cycle, have been "evolving" for thousands of years in virtue of the instantiation of different entities that carry out these processes and keep them ongoing. Doolittle refers to this account as the song (process), not singer (entity) account, for what keeps alive and evolving is the cycle, rather than the organisms that carries it out. He thinks that this type of account helps to make sense of some of the claims made by HTE defenders. I reckon the 
merits of Doolittle's account, and although the song/singer account is an important and original step towards disentangling part of the evolutionary implications of HTE and does so in a similar way than the stability of traits account does so, I think it is very different from it.

On the one hand, the song/singer account is about the patterns of interaction, irrespectively of the lineages that bring these patterns of interaction about. In this sense, the evolution of the host genome turns out to be irrelevant from this perspective: the host genome is merely another singer of the song, but any other host genome could sing the same song. The stability of traits account, on the other, puts the emphasis precisely on the host genome, and aims to trace its evolution in relation to the evolution of the traits encoded in its microbiome. In other words, it aims to explain why a relatively genetically poor host genome (vampire bat genome) can carry out the type of activities it carries out (hematophagy) in virtue of how its functional microbiome has evolved. In that vein, the microbiome is seen as an extension of the host genome, which externalizes part of its resources in the microbiome, and guarantees that these resources (mostly traits, not lineages) will be available transgenerationally.

- $\quad$ The community genetics account of the hologenome. Lloyd and Wade (2019) have recently defended that the holobiont is an evolutionary individual from the perspective of community genetics. In their account, the mistake in part of the hologenome literature is to consider that coevolution takes places between species, rather than between traits. Taking a trait-to-trait perspective, and applying the tools from community genetics, they elaborate a view of the holobiont that supports the thesis that holobionts are evolutionary individuals (units of selection) that are required to understand how host-symbiont associations evolve, including how parasitic symbiosis evolve toward mutualism, or how horizontal transmission evolves towards vertical transmission, etc. Although I think their view is in many senses correct and it provides the best defense of HTE formulated so far, it assumes a completely different ontology of the holobiont than the one that the stability of traits account entails. For Lloyd and Wade, the holobiont is the evolutionary individual that makes that some host-symbiont associations evolve due to the epistatic interactions between the traits in their genomes. They argue that these interactions may sometimes evolve in one-way, e.g. only in the host and not in the microorganisms and use the case of the bobtail squid and Vibrio fischeri to illustrate their position (Lloyd and Wade 2019: 164-166). But they do not explicitly consider the possibility that some of the traits of the microbiome can form an evolutionary individual together with the host genome, and independently of the lineages that bear them, or of 
whether they are coevolving or not with the host genome. ${ }^{22}$ In their view, even though what matters to understand holobiont evolution is trait-to-trait coevolution, the entities that are coevolving (and thus compose the evolutionary holobiont) are the host and the symbiont lineages. In that sense, Lloyd and Wade's account is radically different from the stability of traits account which neither requires trait-to-trait coevolution (although it may be an outcome of selection on the hologenome), nor that a consequence of the interactions the host-symbiont relationship evolves towards mutualism or vertical transmission.

\section{Conclusion}

In this paper, I have argued that Bourrat and Griffiths' fitness boundedness through fitness alignment framework to distinguish between true multispecies individuals and mere multispecies assemblages is consistent with the intuitions that lie behind the part of the system arguments proposed by HTE defenders. My argument has been given in two steps. First, drawing on some recent research about the evolution of sanguivory in vampire bats (Mendoza et al. 2018) I have argued that, adequately refined, part of the system arguments do usually mask an evolutionary fact of the matter (section 3). Drawing on that, I have elaborated a new conception of the boundaries of the evolutionary holobiont that I called "stability of traits" account, according to which the evolutionary individual would be the host genome plus some of the traits in its microbiome. I have argued that the host genome and some of these traits in the microbiome do indeed have their fitness interests aligned, and thus satisfy Bourrat and Griffiths' criterium of fitness boundedness. In this sense, what may be seen as merely metabolic, developmental or immunological arguments (the so-called "part of the system" arguments) do indeed mask an evolutionary fact of the matter that concerns the fitness alignment of the entities that compose the holobiont (section 4).

The view of the holobiont I am advocating is radically different from other views of the holobiont. First, contrary to most HTE defenders, I don't define the evolutionary holobiont as the collection of genomes, but rather and the sum of the host genome and some of the traits of its microbiome. I take that the main lesson from our contemporary research on holobionts is that host lineages could not have evolved alone, and it is important to investigate the kind of evolutionary shifts in their microbiomes to properly understand why they can cope with the type of selective pressures they cope with. In this line, the stability of traits account indicates which parts of the microbiome may have been evolving, and why, without making strong evolutionary assumptions about the collection of lineages (genomes) that make up the holobiont.

\footnotetext{
${ }^{22}$ One reviewer argues that this form of independence seems to entail a form of emergence that rejects reductive physicalism, and that would require separate defence. I defend that view in Suárez and Triviño (2020).
} 
The validity of the stability of traits account strongly depends on the evolutionary history of each host lineage, and each host family, and it is hard to a priori evaluate how strong selection for holobionts has been in each host lineage. What may have happened in the case of vampire bats, which are used to motivate the account, could have been the exception, rather than the rule. Nonetheless, the stability of traits account suggests a new way of exploring holobiont evolution that could shed some new light, and that is worth exploring. And the account can suggest new forms of interpreting phenomena such as the role of the microbiome in speciation (Brucker and Bordenstein 2012, 2013), the evolution of complex lifestyles such as hematophagy (Mendoza et al. 2018), or herbivory (Gilbert 2019), or the acquisition of complex adaptations such as immunological resistance (Reshef et al. 2006), or mating preferences (Sharon et al. 2010). To reinterpret these cases is however outside the scope of this paper.

\section{Acknowledgments}

Different versions of this paper have been presented in the IAS-Research Seminar (University of the Basque Country, 2017), the European Philosophy of Science Association 17 (University of Exeter, 2017), the IX Conference of the Spanish Society for Logic, Methodology and Philosophy of Science (UNED, Madrid, 2018), the International Society for History Philosophy and Social Studies of Biology 2019 (University of Oslo, 2019), and the Congress of Logic, Methodology and Philosophy of Science and Technology 2019 (University of Prague, 2019). I acknowledge all the participants for their comments and feedback. Special thanks to John Dupré, for his constant feedback and encouragement, and Staffan Müller-Wille and Samir Okasha for having discussed these ideas with me in the wider context of my doctoral thesis. Also, thanks to Thomas Bonnin, Seth Bordenstein, Pierrick Bourrat, Mark Canciani, Roger Deulofeu, Ford Doolittle, Scott Gilbert, Cipran Jeler, William Jones, Ehud Lamm, Lisa Lloyd, Lisandra Z. Mendoza, Álvaro Moreno, Maureen O’Malley, Eugene Rosenberg, Elliot Sober, Adrian Stencel, Vanessa Triviño, Davide Vecchi, Sophie Veigl, Ken Waters, and Ilana Zilber-Rosenberg for having read and discussed previous versions of this paper with me. Finally, the Spanish Ministry of Economy and Competitiveness (FFI2016-76799-P), the Spanish Ministry of Education (FFU16/02570), and the Royal Institute of Philosophy are formally acknowledged for their economic support.

\section{References}

Ariew A., and R. C. Lewontin. 2004. The confusions of fitness. Br J Philos Sci 55: 347363. 
Bennett G. M., and N. A. Moran. 2015. Heritable symbiosis: the advantages and perils of an evolutionary rabbit hole. PNAS USA 112: 10169-10176.

Booth, A. 2014. Symbiosis, selection and individuality. Bio Philos 29: 657-673.

Bordenstein SR \& KR Theis. 2015-.Host biology in light of the microbiome: ten principles of holobionts and hologenomes. PLoSBiol 13.8: e1002226.

Bouchard, F. 2013. What is a symbiotic superindividual and how do you measure its fitness. In F. Bouchard, and P. Huneman (eds) From groups to individuals. MIT Press, Cambridge, pp. 243-264.

2014 Ecosystem evolution is about variation and persistence, not populations and reproduction. Biol Theory 9: 382-391.

Bourrat, P. 2019. Evolutionary Transitions in Heritability and Individuality. Theory in Biosciences 138 (2): 305-23. https://doi.org/10.1007/s12064-019-00294-2.

Bourrat, P., and P. Griffiths. 2018. Multispecies individuals. History and Philosophy of the Life Sciences 40(2): 33.

Brooks AW, Kohl KD, et al. 2016. Phylosymbiosis: relationships and functional effects of microbial communities across host evolutionary history. PLoSBiol 14 (11): e2000225

Brucker, R. and S. Bordenstein. 2012. Speciation by Symbiosis. Trends Ecol Evol 27 (8): 443-451

Brucker, R. and S. Bordenstein. 2013. The hologenomic basis of speciation: Gut bacteria cause hybrid lethality in the genus Nasonia. Science 341: 667-669.

Brucker, R. and S. Bordenstein. 2014. Response to Comment on "The hologenomic basis of speciation: gut bacteria cause hybrid lethality in the genus Nasonia”. Science 345(6200): 1011-1011.

Clarke, E. 2013. The multiple realizability of biological individuals. The Journal of Philosophy CX(8): 413-435.

Díaz, J. S. 2015. El mecanismo evolutivo de Margulis y los niveles de selección. Contrastes. Revista internacional de filosofía XX(1): 7-26.

DiFrisco, J. 2017. Kinds of biological individuals: Sortals, projectability, and selection. The British Journal for the Philosophy of Science 70(3): 845-875.

Doolittle, W. F. 2017. Darwinizing Gaia. J Theor Biol 434: 11-19.

Doolittle, W. F., and A. Booth. 2017. It's the song not the singer: an exploration of holobiosis and evolutionary theory. Biol Philos 32(1): 5-24.

Doolittle, W. F., and S. A. Inkpen. 2018. Processes and patterns of interaction as units of selection: An introduction to ITSNTS thinking. PNAS USA 115(16), 4006-4014. 
Dupré, J. 2010. The polygenomic organism. The Sociological Review 58(s1): 19-30. . 2012. Processes of life: Essays in the Philosophy of Biology. Oxford. Oxford University Press.

. 2017. The metaphysics of evolution. Interface focus 7: 20160148.

http://dx.doi.org/10.1098/rsfs.2016.0148

Dupré, J., and M. A. O’Malley. 2009. Varieties of living things: Life at the intersection of lineage and metabolism. Philosophy \& Theory in Biology 1 (December).

Douglas, A. E., and J. H. Werren (2016) Holes in the hologenome: why host-microbe symbioses are not holobionts. mBio 7(2): e02099-15.

Gilbert, S. F. 2019. Developmental symbiosis facilitates the multiple origins of herbivory. Evolution \& Development. https://doi.org/10.1111/ede.12291

Gilbert, S. F., J. Sapp, and A. I. Tauber. 2012. A symbiotic view of life: We have never been individuals. The Quarterly Review of Biology 87(4): 325-341.

Gilbert, S. F., E. Rosenberg, and I. Zilber-Rosenberg. 2017. The holobiont with its hologenome is a level of selection in evolution. In S. B. Gissis, E. Lamm, and A. Shavit (eds.) Landscapes of collectivity in the life sciences. London. The MIT Press. (pp. 305324).

Godfrey-Smith, P. 2009. Darwinian populations and natural selection. Oxford NY. Oxford University Press.

10120-10125.

. 2015. Reproduction, symbiosis, and the eukaryotic cell. PNAS 112(33):

Griffiths, P. E., and K. Stotz. 2013. Genetics and Philosophy: An Introduction.

Cambridge: Cambridge University Press.

Hester ER, KL Barott, et al. 2016. Stable and sporadic symbiotic communities of coral and algal holobionts. The ISME Journal 10:1157-1169

Hull, D. L. 1980. Individuality and Selection. Annual Review of Ecology and Systematics 11: 311-332.

Hurst GDD. 2017. Extended genomes: symbiosis and evolution. Interface Focus 7: 20170001

Jefferson, R. 1994. The Hologenome. Agriculture, Environment and the Developing World: A Future of PCR. Cold Spring Harbor, New York.

Kushmaro, A., E. Rosenberg, M. Fine, et al. 1997. Bleaching of the coral Oculina patagonica by Vibrio AK-1. Mar Ecol Prog Ser 147: 159-165.

Kushmaro, A. E. Banin, Y. Loya, et al. 2001. Vibrio shiloi sp. nov., the causative agent of bleaching of the coral Oculina patagonica. International Journal of Systematic and Evolutionary Microbiology 51: 1383-1388. 
Lamm, E. 2018. Big Dreams for Small Creatures: Ilana and Eugene Rosenberg's path to the Hologenome Theory. In O. Harman, and M. R. Dietrich (eds.) Dreamers, visionaries, and revolutionaries in the life sciences. University of Chicago Press.

Lerner A., T. Matthias, and R. Aminov. 2017. Potential Effects of Horizontal Gene Exchange in the Human Gut. Front Immunol 8: 1630.

Lemanceau, P., M. Blouin, D. Muller, and Y. Moënne-Loccoz. 2017. Let the core microbiota be functional. TRENDS in Plant Science 22(7): 583-595.

Lidgard, S., and Nyhart, L. K. 2017. The work of biological individuality: Concepts and contexts. In S. Lidgard and L. K. Nyhart (eds.) Biological Individuality. Integrating scientific, philosophical, and historical perspectives. Chicago. The University of Chicago Press.

Liu, L., X. Chen, G. Skogerbø, et al. 2012. The human microbiome: a hot spot of microbial horizontal gene transfer. Genomics 100(5): 265-270.

Lloyd, E. 2017a. Units and Levels of selection. In E. N. Zalta (ed.) Stanford Encyclopaedia of Philosophy. https://plato.stanford.edu/entries/selection-units/

Lloyd, E. 2017b. Holobionts as units of selection: Holobionts as interactors, reproducers, and manifestors of adaptation. In S. B. Gissis, E. Lamm, \& A. Shavit (Eds.), Landscapes of Collectivity (Vienna Series in Theoretical Biology). Cambridge, MA: MIT Press.

Lloyd, E., and M. J. Wade. 2019. Criteria for holobionts from community genetics. Biol Theor. https://doi.org/10.1007/s13752-019-00322-w.

Louca, S., S. M. S. Jacques, A. P. F. Pires, J. S. Leal, D. S. Srivastava, L. W. Parfrey, V. F. Farjalla, and M. Doebeli. 2016. High taxonomic variability despite stable functional structure across microbial communities. Nat Ecol Evol 1: 15.

Lu, Q., and P. Bourrat. 2017. The Evolutionary Gene and the Extended Evolutionary Synthesis. British Journal for Philosophy of Science 69(3): 775-800.

doi:10.1093/bjps/axw035.

Margulis, L. 1990. Words as battle cries: Symbiogenesis and the new field of endocytobiology. Bioscience 40(9): 673-677.

. 1991. Symbiogenesis and symbioticism. In L. Margulis, and R. Fester (eds.) Symbiosis as a source of evolutionary innovation. London. The MIT Press. (pp. 1-14)

Margulis, L., and D. Sagan. 2001. The beast with five genomes. Natural history 110(5): 38-41.

Maynard-Smith, J. 1987. Evolutionary progress and levels of selection. In: Dupré, J. (ed.) The latest on the best: essays on evolution and optimality. MIT Press. Cambridge. . 1991. A Darwinian view of symbiosis. In: Margulis, L., and Fester, R. (eds.) Symbiosis as a source of evolutionary innovation. The MIT Press. Cambridge. 
Mendoza, M. L. Z., Z. Xiong, M. Escalera-Zamudio, et al. 2018. Hologenomic adaptations underlying the evolution of sanguivory in the common vampire bat. Nature Ecol Evol 2: 659-668.

Moran, N., and D. B. Sloan. 2015. The Hologenome Concept: Helpful or Hollow? PLoSBiol 13(12): e1002311.

Okasha, S. 2006. Evolution and the levels of selection. Oxford. Oxford University Press. O’Malley, M. A. 2017. From endosymbiosis to holobionts: Evaluating a conceptual legacy. J Theor Biol 434: 34-41.

Pound, R. 1893. Symbiosis and mutualism. Am Nat 27(318): 509-520.

Pradeu, T. 2016. The many faces of biological individuality. Biol Philos 31: 761-773.

Queller, D. C., and J. E. Strassmann. 2016. Problems of multispecies organisms: endosymbionts to holobionts. Biol Philos 31: 855-873.

Reshef, L., O. Koren, I. Zilber-Rosenberg, et al. 2006. The coral probiotic hypothesis. Environ Microbiol 8(12): 2066-2073.

Rohwer, F., V. Seguritan, F. Azam, et al. 2002. Diversity and distribution of coralassociated bacteria. Mar Ecol Prog Ser 243: 1-10.

Rosenberg, E., and I. Zilber-Rosenberg. 2013. The Hologenome Concept. London. Springer.

2016. Microbes drive evolution of animals and plants: the hologenome concept. mBio 7(2): e01395-15.

Roughgarden, J., S. F. Gilbert, E. Rosenberg, I. Zilber-Rosenberg, and E. Lloyd. 2018. Holobionts as units of selection and a model of their population dynamics and evolution. Biological Theory 13(1): 44-65.

Roughgarden, J. 2019. Holobiont Evolution: Mathematical Model with Vertical vs. Horizontal Microbiome Transmission. bioRxiv. https://www.biorxiv.org/content/10.1101/465310v5

Schneider, A. 1897. The phenomena of Symbiosis. Minnesota Botanical Studies 1(9): 923-948.

Sharon G., D. Segal, J. Ringo, et al. 2010. Commensal bacteria play a role in mating preference of Drosophila melanogaster. PNAS USA 107(46): 20051-20056.

Skillings, D. 2016. Holobionts and the ecology of organisms: Multi-species communities or integrated individuals? Bio Philos 31: 875-892.

Stencel, A. 2016. The relativity of Darwinian populations and the ecology of endosymbiosis. BioPhilos 31(5): 619-637. 
Stencel, A. Forthcoming. Why the evolution of heritable symbiosis neither enhances nor diminishes the fitness of a symbiont? PTPBio.

Stencel, A., and A. Proszewska. 2017. How research on microbiomes is changing biology: A discussion of the concept of the organism. Foundations of Science 23(4): 603-620.

Stencel, A., and D. M. Wloch-Salamon. 2018. Some theoretical insights into the hologenome theory of evolution and the role of microbes in speciation. Theory Biosci. 137(2): 197-206. https://doi.org/10.1007/s12064-018-0268-3

Suárez, J. 2016. Bacterial species pluralism in the light of medicine and endosymbiosis. Theoria 31(1): 91-105.

Suárez, J. 2018. The importance of symbiosis in philosophy of biology: An analysis of the current debate on biological individuality and its historical roots. Symbiosis 76(2): 77-96.

Suárez, J. 2019. The Hologenome Concept of Evolution: A Philosophical and Biological Study. PhD Disseration. Exeter: University of Exeter. http://hdl.handle.net/10871/39349.

Suárez, J., and V. Triviño. 2019. A metaphysical approach to holobiont individuality: Holobionts as emergent individuals. Quaderns de Filosofia 6(1): 59-76.

Suárez, J., and V. Triviño. 2020. What is a hologenomic adaptation? Emergent individuality and inter-identity in multispecies systems. Frontiers in Psychology 11: 187.

Taxis T. M., S. Wolff, S. J. Gregg, et al. 2015. The players may change but the game remains: network analyses of ruminal microbiomes suggest taxonomic differences mask functional similarity. Nucleic Acids Research 43(20): 9600-9612.

Theis K. R., N. M. Dheilly, J. L .Klassen, et al. 2016. Getting the hologenome concept right: an ecoevolutionary framework for hosts and their microbiomes. mSystems 1(2): e00028-16.

Vieira-Silva, S., G. Falony, Y. Darzi, G. Lima-Méndez, R. García Yunta, S. Okuda, et al. 2016. Species-function relationships shape ecological properties of the human gut microbiome. Nature Microbiology 1: 1-8.

Vrba, E. and J. S. Gould. 1986. The hierarchical expansion of sorting and selection: Sorting and selection cannot be equated. Paleobiology 12 (2): 217-228.

Zilber-Rosenberg, I. and E. Rosenberg. 2008. Role of microorganisms in the evolution of animals and plants: the hologenome theory of evolution. FEMS Microbiol Ecol 32.5: 723-735. 